\title{
CRITICAL ISSUES IN STRENGTH EVALUATION OF TURBO MACHINERY BLADE DISK ASSEMBLY AT CONSTANT RPM
}

\author{
Kumar $K^{1}$., Ajit Prasad S.L ${ }^{2}$., Ramachandra K. ${ }^{3}$ \\ ${ }^{1}$ Research scholar, PES College of Engineering, Mandya, India \\ ${ }^{2}$ Department of Mechanical Engineering, PES College of Engineering, Mandya, India \\ ${ }^{3}$ Gas Turbine Research Establishment, Bangalore, India \\ Email: kumar.triveni@gmail.com
}

\begin{abstract}
In this present work, 3D Finite Element Analyses (FEA) of Low Pressure (LP) steam turbine bladed disk assembly are carried out at a constant speed loading condition. The prime objective of this work was to optimize the geometry of bladed disk root with the aid of Peterson's Stress Concentration Factor (SCF) charts available in literature. Secondly design rules are developed for structural integrity of blades and disc considering a common factor for material, manufacturing and temperature uncertainties.These design rules are in turn used as design checks with aid of finite element analysis results. Special investigations were been performed based on Neuber formulae for reducing the local peak stresses at blade and disc root fillet using linear Analysis to identify the equivalent non linear stress value by strain energy distribution method for estimating the minimum no of cycles required for crack initiation by Low Cycle Fatigue (LCF) calculations
\end{abstract}

Key words: Please provide a minimum of 5 and a maximum of 7 keywords related to the subject of the article

\section{INTRODUCTION}

The importance of power generation through thermal and nuclear plants is very much felt due to the even fluctuations in monsoon, which has made the power generation and supply inconsistent. With the ever-increasing demand for power due to increased industrial activities, it has become imperative to generate power economically and consistently. One such attempt has resulted in increased use of steam turbine for power generation. In a steam turbine, a number of blades are used for the energy transfer. These blades are subjected to centrifugal force and these blades are the critically important parts which affecting the satisfactory function of a turbine. Bladed disk are most flexible elements in high speed rotating machinery. Due to rotation, the blade root gets tightened in the disk slot and transmits the centrifugal load. The mating contact may be just two or may increase to six or more for low-pressure blades. While the average stress in the mating surface is fully elastic and well below yield, the peak stress at singularities in the groove shape can reach yield values and in to local plastic region. Last stage LP turbine blades are the most severely stressed blades in the system. Usually these are the limiting cases of blade design allowing the peak stress to reach yield or just below yield conditions. Failure can occur with the crack initiation at the stress raiser location and propagation, two cases can be cited. Now DOE techniques and optimization methods have become available to optimize the shape and minimize the peak stress values so as to improve the structural integrity of bladed disk. Because the problem is highly nonlinear due to centrifugal stiffening and spin softening, considerable time is taken to achieve the optimized root with the application of Peterson's Stress concentration chart [1].There is a vast literature on linear-elastic stress concentration factors Kt (Peterson, 1997), which depend solely on the specimen/notch geometry and on the type of loading. However, in the presence of plasticity at the notch root, the actual stress concentration factor $K \sigma$ is found to be smaller than the tabulated Kt, mainly due to the stress redistribution at the yielding zone. In turn, the strain concentration factor $K \varepsilon$ at the notch root, which strongly affects the fatigue life predicted by the $\varepsilon N$ method, can be much larger than Kt. Several models have been proposed to assess the elastic-plastic behavior at the notch root, such as the rules proposed by Neuber (1961), Glinka (1985), Topper et al. (1969), Seeger et al. (1980), and Hoffman et al. (1985). In many cases, these models may provide reasonable estimates of the maximum stresses and strains at the notch root; however, the differences among the fatigue life predictions by each rule can be unacceptably large. In addition, these methods do not 
account for the geometrical changes at the notch root under large displacements, leading to further errors.

With the light of the above the present work is related to low pressure stage blades of steam turbine. Usually these blades are subjected to failure due to huge centrifugal stresses and low flow. The present work centrifugal forces were considered as main cause for the failure. Therefore estimation of peak stress becomes important in deciding the minimum no of cycles required for crack initiation in both blade as well as in disc. Here one such effort was made to perform linear stress analysis to reduce the local peak stresses by modifying the blade root fillet along with parametric modification in disc based on application of Petersons stress concentration factor chart and analytical verification. Further work was extended to understand the various design parameters involved in deciding the safety margins for structural integrity of bladed disc assembly.

\section{OBJECTIVES OF THE PRESENT WORK}

The main objective of this study was to carry out the structural analyses of LP stage blade disc sector for its strength evaluation. 1. Develop a design rule for turbo machinery blades that ensures structural requirement 2. Effective utilization of design rule to meet the structural requirements in par with Finite element results as a design check 3 . To modify the blade root to reduce the stress concentration at root and modify the disc corresponding to the modified blade root dimension as per Peterson's SCF charts.

\section{BLADE TYPE}

Following the steam path through a turbine the environment for the energy converging blades varies strongly and, as a consequence, so do the mechanical requirements. These requirements have a strong influence on the choice of material and the design with respect to temperature, wetness and cleanliness of medium, acting forces and other factors as i.e. harden ability and oxidation. Therefore, different blade families exist which can be categorized according to their use in the primary three turbine modules as high, intermediate and low-pressure blades (HP, IP and LP). Generally the LP stage blades are shrouded or laced, because they have to resist huge centrifugal forces and bending produced by the steam loads. Fig. 1 shows the geometry of the LP blade.

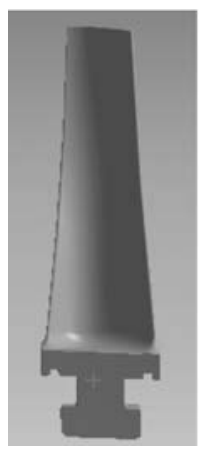

Fig. 1. Simple 'T' root LP Blade

\section{DESIGN RULE DEVELOPMENT AND USE}

\section{A. Factor of Safety}

Based on the level on uncertainty in material property i.e. grain boundary distribution, manufacturing uncertainties like variable transition fillet radius at base of airfoil both at LE,TE and at chord cover. Over speed of $11 \%$ and the control system to break the separation margins and design speed and over speed condition, uncertainty due to temperature variation and no of over speed in one start up and shut down cycle all three important parameters should be given a common factor which goes in to the margin limits at minimum yield of the material as 'factor of Safety'

$$
\sigma_{\text {allowable }}=\frac{\sigma_{\text {minimum }}}{(\text { oversped })^{2} *(\text { additionalFOS })}
$$

Since speed plays a vital role in rotary machinery and centrifugal force $F c=m \omega^{2} r$ special care to be provided for speed load which varies as a square function. Factors for temperature, manufacturing and material uncertainty is taken $15 \%$ over minimum yield at designed speed. At designed speed, factor of safety $=(1.212)^{*}(1.15)=1.68$ allowable FOS at different speeds is as shown in table 1.0

Table 1. Factor of Safety limits

\begin{tabular}{|c|c|}
\hline Speed & Allowable FOS limits \\
\hline $100 \%$ & 1.68 \\
\hline $110 \%$ & 1.18 \\
\hline $121 \%$ & 1.15 \\
\hline
\end{tabular}

This indicates that the material has $15 \%$ additional margin at over speed condition. The allowable average stress limit at average stage temperature of $750^{\circ} \mathrm{C}$ for this blade with a Minimum yield of $585 \mathrm{MPa}$ is $349 \mathrm{Mpa}$. 


\section{AVERAGE SECTION STRESS}

It is seen that low-pressure blades are highly subjected to centrifugal load and thermo mechanical loads. Since the centrifugal load dominates the thermal loads for every mission cycle emphasis is paid to address mechanical loads for structural integrity of blades. In bladed disc assembly the blade happens to be a weaker section compared to disc since replacing blades under failure to much cheaper than disc. Average section stresses attained at minimum cross section at operating and over speed condition should be with in the allowable design limits both in blades and disc. The design rule is the average section stress in the disc groove should have greater margin compared to blades, based on weak link analogy.

The critical zones identified in a bladed disc assembly.

Average section stress in airfoil should be less than allowable stress limits at design speed.

Average section at blade root neck and at minimum cross-section of disc should be within the allowable design limits.

Average section stress in minimum cross section of disc should be $10 \%$ less compared to blade root average section stress based on weak link analogy.

Average section stress in the disc considering loading and unloading characteristics at over speed conditions should have a margin 1.18 of $0.2 \%$ proof stress

AWAHS(area weighted average hoop stress) in disc neglecting the stress concentration zones should have a margin greater than 1.22 at a over speed of $122 \%$ of operating speed since disc is sensitive to hoop burst at bursting speed separating correction factors should be induced for variation of thermal gradients from bore to rim. The amount of plastic strain accumulated at bore is the deciding factor for margin evaluation. the amount of residual stress developed in disc for every mission cycle decides the low cycle fatigue life of the disc hence to make the design criteria simple at over speed condition $0.2 \%$ proof stress is considered for evaluation of over speed margin and ultimate tensile stress is considered for burst speed margin at most care should be taken to choose the Experimental data for elastic properties at appropriate temperature for notched specimens. Airfoils in recent years are designed with taper, twist good camber sufficient chord length, greater LE and TE thickness along with generous transition radius to avoid the stress concentration at base of airfoil. the average section stress from hub to tip should be designed in descending order with in the allowable design limits to avoid gross yielding at operation speed

\section{LOW CYCLE FATIGUE LIFE APPROACH}

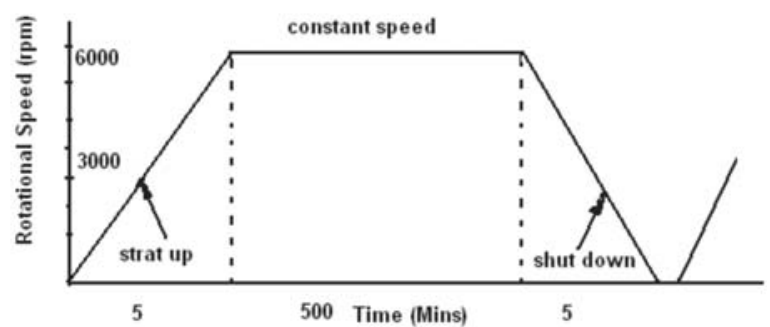

Fig. 2. Blade Loading Pattern

The blade loading pattern is as shown in Fig 2.0. Often the blade root fillets and disc groove fillets undergo highest peak stress/strain for every cyclic loading (start up cycle). Therefore some amount of plastic strain gets accumulated for every start up and shut down cycle. Therefore the total strain accumulated. $=$ Sum of (total elastic + total plastic) based on the desired amount of start up and shut down cycles required for a given component a common factor should be considered like factor of safety for manufacturing and thermal uncertainties to decide upon the minimum no of start up and shut down cycles required to initiate the crack in blade if the blade has to have a minimum of 10000 cycle (Start/shut down) it can be designed with a knock down factor (KDF) of 2 for 20000 cycles. Looking at the $\varepsilon N$ curve data for a given material the total strain may be considered independently for lifing the blade and disc based on the strength of the material for local strain approach. For stress based approach the linear stress may be neubraised using re-distribution of strain energy under the stress-strain curve applying Neuber's rule the same required no of design cycles can be achieved. By co-relating linear stress levels with obtained total strain required to achieve the minimum start up shut down cycles. In the present work one such effort was promptly made to reduce the peak stresses to improve LCF life of blade disc assembly. The stress base theory, which has been recognized as much slower in modeling the crack initiation phase, the most promising approach seems to be based on this approach. Although the crack being not initiated in the model, the 
life of the component before the crack initiates is our concern. The basic premises is that the local fatigue response of the material at the critical point i.e., at the site of crack initiation, is analogous to the fatigue response of a component subjected to cyclic stresses to properly perform the local cyclic stress strain history at the critical point in the structure must be determined either by analytical or experimental means. Therefore a valid cyclic symmetry pre stress static analysis procedure is carried out using finite element modeling or experimental strain measurements. It must be recognized that the phenomena of cycle dependent stress relaxation as well as sequential loading effect and residual stress effects may be experienced by the specimen as it accumulates fatigue damage presumed to be the same as at the critical point.

\section{FINITE ELEMENT MODEL}

For the present analysis a cyclic symmetry sector of a bladed disk assembly of the LP stage was considered. The geometry of the one blade with disc sector was modeled using commercially available modeling software Pro-E. The finite element model of LP sector stage bladed disc is as shown in Fig.3. For the present analysis SOLID45 element was considered to generate the finite element model. Care was taken to make sure there is not degenerated tetrahedral elements exist in the mesh, as the de-generated Tet elements are not good for accurate stress calculation. Surface to Surface contact pairs were created between the blade and disk mating surfaces. A matching node pattern was maintained at the blade root and disk hook pressure faces (in order to avoid any ambiguities on stress values due to contact elements), where the loads transfer between blade and disc takes place. Cyclic boundary conditions were applied on both the partition surfaces The blade and disc both are assumed to be
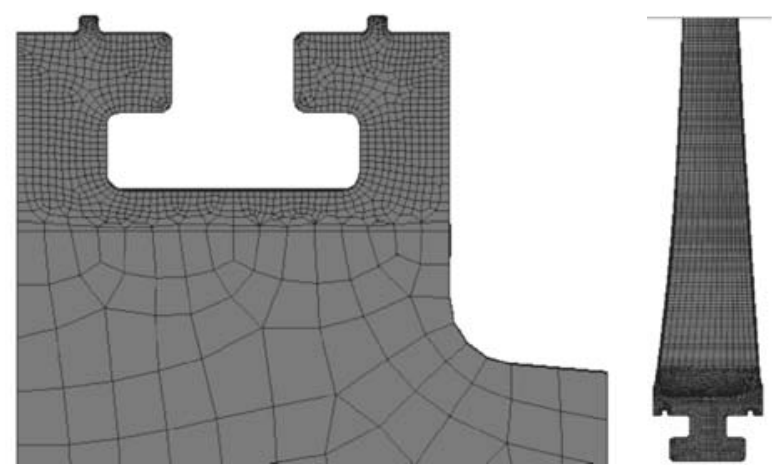

Fig. 3. FE Model of Blade Disc Sector made up of same material with yield stress of 585 $\mathrm{MPa}$, Young's modulus 210Gpa, density $7900 \mathrm{Kg} / \mathrm{m}^{3}$ and Poison's ratio 0.3.

\section{A. Base Line Model:}

The peak Von-Mises stress computed in fig $4 \& 5$ were much higher than the yield stress (585 MPa) of the material. Hence the use of linear analysis was alarming. However, the traditional nonlinear FEA analysis simulating the local material plasticity are still very resource intensive, yet fatigue and life endurance simulations commonly need stress and strain results for various different load levels, making such an analysis expensive. In order to reduce the number of nonlinear simulation results, approximation techniques based on the Neuber's formula [9] which estimate the plastic stress-strain state from linear analysis runs were utilized. With the application of Finite Element Analysis, notch concentration factors are being inherently considered. The general Neuber's procedure of extrapolating linear stresses into the plastic material region can thus is applied to arbitrary geometries. The further discussed comparisons and design decision
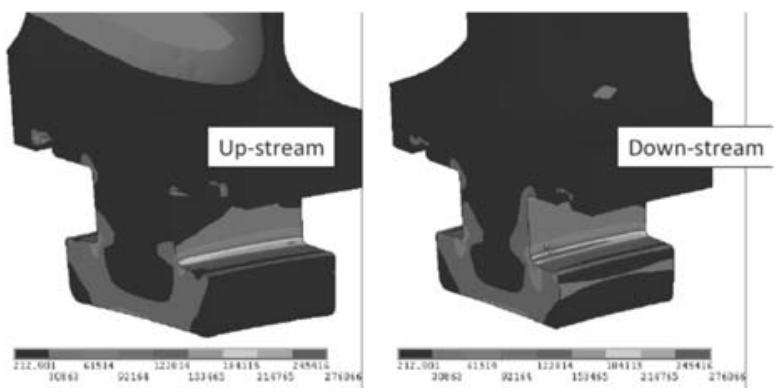

Fig. 4. Von-Mises stress in elastic domain at 6000 RPM

Peak stress of $1904 \mathrm{MPa}$ is seen at Blade root, Average stress of $250 \mathrm{MPa}$ at Blade neck

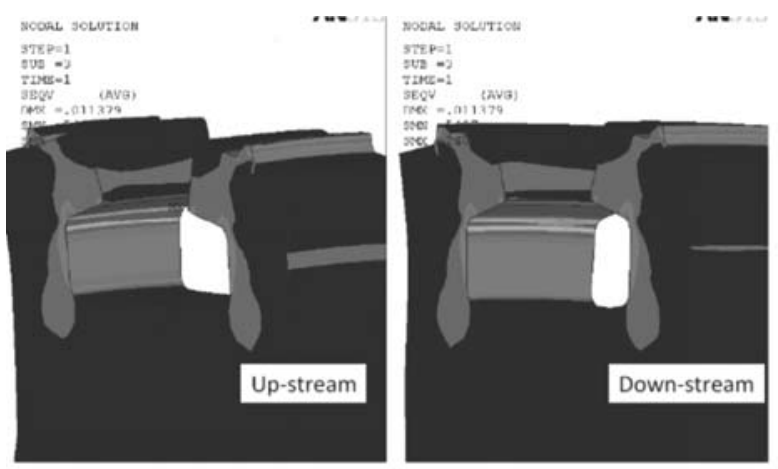

Fig. 5. Von-Mises stress in elastic domain in Disc at 6000 RPM 


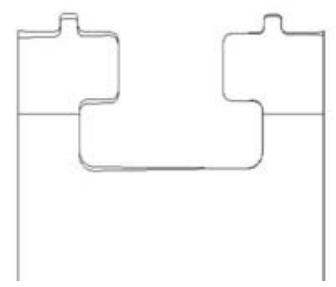

Fig. 6. Average stress at minimum cross-section

Peak stress of $1660 \mathrm{MPa}$ is seen at Blade root are based on stresses after Neuber's procedure [8].The base line geometry shows a peak stress of $1904 \mathrm{MPa}$ in blade root fillet, it lead to very poor LCF cycles [9], calculated based on the stress from Neuber's formula. Also the knowledge based design criteria (established based on experience and experimental tests) limits the peak stress to $1200 \mathrm{MPa}$ from the linear finite element stress analysis, for the component life of 5000 start up/shut down cycles. Since the base line model shown peak stress of $1904 \mathrm{MPa}$ from linear stress analysis, optimization of blade root was required to bring down the peak stress.

\section{B. Design checks}

How ever the design rule for Average Section stresses (a), (b), (c), d) in Airfoil, blade root and disc groove minimum cross-section was well with in the allowable design limits of $349 \mathrm{MPa}$. At design speed .The necessary design margins at over speed conditions with Radial growth at average stage temperature of $700^{\circ} \mathrm{C}$ as per design rule was satisfied, but except for the local peak stress/strain at the blade root fillet which was not sufficient to achieve the minimum desired LCF life cycle. The radial growth of blade at operating conditions is well within the clearance of $3 \mathrm{~mm}$ between blade tip and casing. This is indicating that there is no rubbing of blade with the casing even at over speed conditions.

\section{Optimization through Peterson's Stress Concentration factor chart}

Experiences show that in order to improve the LCF life of a bladed disk assembly, a corrective measure usually involves a design or geometry modification of the blade root section where the peak stress occurs due to change in geometry. By studying stress concentration factors at the blade root much can be learned about how to produce designs, that is superior from stand point of resistance to repeated loads and how to evaluate approximately the influence of various geometric features. The chart from
Peterson's book [1] was used as an "edge finder" [3] to reduce the SCF. Blade root geometry dimension was modified to have theoretical SCF of 6 where as the base line geometry has SCF of 10.4. The modified geometric model was then analyzed using ANSYS to study the sensitivity of the modified geometry. The modification of the blade root is shown in Table 2.0 and the different parameters in Table 2.0 were illustrated in Fig.7.

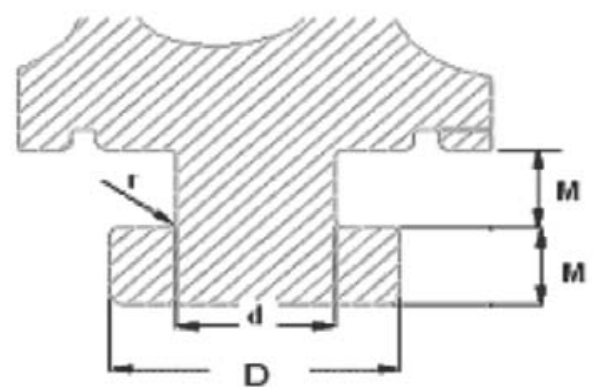

Fig. 7. Root terminology
$r$ - Blade root radius
$L$ - Contact length
D - Land width
M - Land height
W - Disc width
d - Blade neck

The modified blade root geometry resulted in peak stress value of $1153 \mathrm{MPa}$ as shown in Fig. 8, from $1904 \mathrm{MPa}$ at blade root fillet, in a similar manner the peak stress in disk hook reduced to $1102 \mathrm{MPa}$ as shown in Fig. 9, from $1660 \mathrm{MPa}$.

Table 2. Root Terminology

\begin{tabular}{|l|c|c|c|c|c|c|c|}
\hline \multicolumn{7}{|c|}{ Dimensional Details of blade root } \\
\hline & d & D & m & r & r/d & L & W \\
\hline $\begin{array}{l}\text { Base } \\
\text { line root }\end{array}$ & 22 & 40 & 13 & 1 & 0.04 & 6 & 65 \\
\hline $\begin{array}{l}\text { Modified } \\
\text { root }\end{array}$ & 22 & 45 & 14 & 2.2 & 0.1 & 7 & 77 \\
\hline
\end{tabular}

From gross yielding point of view the average stress in blade neck was 242 MP with a FOS of 1.68 at minimum yield at room temperature.

The same exercise was repeated for $121 \%$ over speed condition of $7200 \mathrm{rpm}$. At this over speed condition, induced stresses were under satisfactory limit for required LCF life the modified blade root did not differ from average stress point of view, over-speed margin or radial growth since not much change in the 
resisting area at the blade root neck or in disc as well at the airfoil. The vibration characteristics assessment of the bladed disk assembly plays a vital role in evaluating structural integrity of the system. This present study limits its scope only with static stress analysis
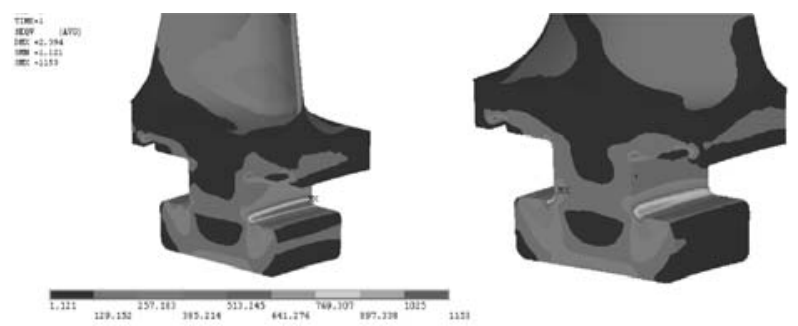

Fig. 8. Von-Mises stress in elastic domain in blade root at $6000 \mathrm{RPM}$

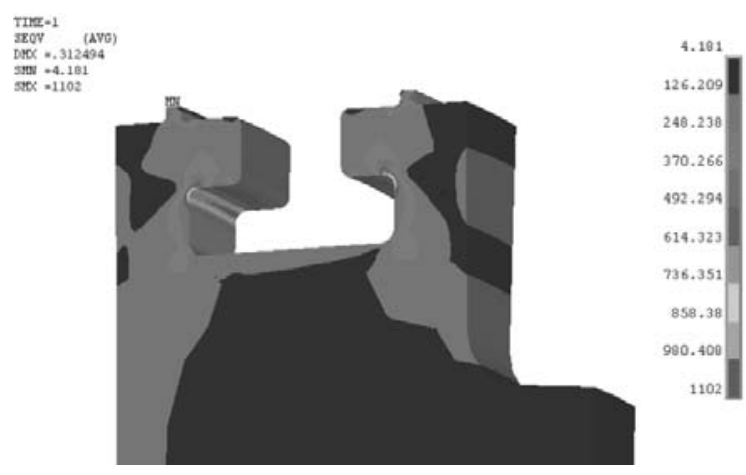

Fig. 9. Von-Mises stress in elastic domain in Disc at 6000 RPM

\section{RESULTS AND DISCUSSIONS}

However; in dynamics point of view vibratory margins have to be accessed by doing a dynamic analysis using cyclic symmetry modal analysis considering the effect of stress stiffening and spin softening by plotting Campbell diagram and Goodman diagram, etc,

In this work the following observations were made pertaining to the LP bladed disc assembly of steam turbine at full speed of 6000rpm with appropriate boundary conditions as explained earlier.

For the base line model linear stress analysis approach shown a peak stress of $1904 \mathrm{MPa}$ in blade root fillet and $1660 \mathrm{MPa}$ at disk hook fillet, when Neuberised lead to very poor LCF life. From gross yielding point of view the average stress in blade neck was with in the desired allowable limit.
The blade root fillet section was strengthened by providing the generous fillet at root. The modifications were made based on Peterson's SCF chart for given $\mathrm{r} / \mathrm{d}$ ratio subjected to tensile loading. This modification successfully brought down stress concentration and improved the LCF life of the component.

The design rule developed can successfully be utilized to provide the structural integrity in turbo machinery blades, disc. These rules act as design check s for verifying the Finite element Analysis results at various design points like Average section stress, over speed margin and radial growth.

\section{CONCLUSIONS}

The following conclusions could be drawn from the present work.

(a) This present work gives a methodological procedure for strength evaluation of a LP steam turbine bladed disk assembly using FEA results and classical approach.

(b) Considering suitable correction factor for design, manufacturing and temperature uncertainties a FOS was evaluated at various speeds

(c) The average section stresses at airfoil, blade root and disc groove is well with in the allowable design and over-speed limits

(d) Based on Peterson's Chart the stress concentration factor was minimized and local strain approach was used to achieve the minimum no of start up and shut down cycles required before crack initiation. Considering one over speed for every start up and shut down cycle

(e) The developed design rule was successfully used in understanding and evaluating the structural requirements and design considerations to be incorporated during the design of turbo machinery components.

\section{REFERENCES}

[1] Pilkey, Walter D, 1997 "Peterson's Stress Concentration Factors". John Wiley \& Sons.

[2] Rao, J.S., 2004, "Transient Stress Analysis and Fatigue Life Estimation of Turbine Blades", Journal of Vibration and Acoustics, ASME, Vol.126, Pp.485- 495. 
[3] Kearton, W J., 1988 "Steam Turbine Theory and Practice", CBS Publishers and Distributors, New Delhi, Seventh Edition.

[4] Christopher-Hermann Richter., 2003, "Structural Design of Modern Steam Turbine Blades Using ADINA", Jou. of Computers and Structures, Elsevier Ltd, Vol. 81, pp.919-927.

[5] Heinz P.Bloch., 1982 "A Practical Guide to Steam Turbine Technology", Mcgraw, Hill.

[6] Rao, J.S., 2000 "Turbine Blade Life Estimation", Narosa Publishing House.

[7] Rao, J.S., 1991 "Turbomachine Blade Vibrations", New Age International $(P)$ Ltd., Publishers, New Delhi.
[8] Neuber. H, 1961 "Theory of Stress Concentration for Shear-Strained Prismatical Bodies with Arbitrary Nonlinear Stress-Strain Law", ASME Journal of Applied Mechanics 28.

[9] Jean Lemaitre, Rodrigue Desmorat, 2005 "Engineering Damage Mechanics: Ductile, Creep, Fatigue and Brittle Failures", Springer .

[10] Hyo-Jin Kim., 1998, "Fatigue Failure Analysis of Last Stage Blade in A Low Pressure Steam Turbine", Engineering Failure Analysis, Elsevier Ltd, Vol.5, pp. 93-100.

[11] Pereira, J.C. et.al, 2007 "A Low Cycle Fatigue Analysis on a Steam Turbine Bladed Disk", 12th IFToMM World Congress, Besançon, June 18-21. 